\title{
Driving advisement with the Elemental Driving Simulator (EDS): When less suffices
}

\author{
ROSAMOND GIANUTSOS \\ Cognitive Rehabilitation Services, Sunnyside, New York
}

\begin{abstract}
The Elemental Driving Simulator (EDS) is a PC-based software and hardware system for advising people with known or suspected cognitive impairment. It is elemental in its simplicity and in its simulation of the elements of driving-related cognitive abilities. The EDS explicitly addresses metacognition and quantitatively relates it to performance. A neuropsychological case example, with an EDS Personal Report, is complemented by statistical findings from (1) workingage drivers (norms), (2) patients with central nervous system impairment seeking driver rehabilitation, and (3) a large sample of older drivers. The EDS proved to be a challenging procedure that all the normals completed more consistently, efficiently, and accurately than the other groups. Clinically, it discriminated extreme cases and, for those who fell in between, it helped focus the issues for further assessment and intervention. The discussion addresses how much realism is necessary, as well as the psychometric limitations of on-road testing.
\end{abstract}

Cognitively at-risk drivers, including the elderly, persons with certain developmental disabilities, and persons recovering from brain injuries, often lack sufficient knowledge and metacognitive (self-appraisal) abilities for evaluating their own capacity for safe driving. Simulation not only permits assessment of these abilities, but also helps demonstrate the findings to the individual. The Elemental Driving Simulator (EDS) is a PC-based driving simulator which explicitly evaluates metacognition and relates it to performance. The EDS procedure is, therefore, designed to help neuropsychologists and others offer driving advisement-objective, individual-specific information and counsel regarding driving.

The EDS grew out of a cognitive and neuropsychological analysis of driving that emphasizes the importance of simultaneous information processing, the ability to deal with complexity, mental flexibility, and impulse control. All of these processes must be evaluated in real time-a requirement for which the PC is ideally suited. Further, clinicians express great concern about drivers' judgments, which can be captured in some degree by the appropriateness of their self-appraisals. Gianutsos, Campbell, Beattie, and Mandriota (1992) have described details of this analysis, the existing approaches in rehabilitative driver assessment, and the Driving Advisement System (DAS)-a precursor of the EDS.

The EDS is also designed to be practical with respect to both time and expense. It requires approximately

The EDS was developed by the author with programming by Aaron Beattie. It is disseminated by Life Science Associates, 1 Fenimore Road, Bayport, NY 11704; 516-472-2111, which pays an author's royalty. Great appreciation is due Amy Campbell, OTR/L and her colleagues at Gaylord Hospital in Wallingford, CT, and to the ITT Hartford Insurance Company for supplying EDS and other data about the rehabilitation patients and older drivers, respectively. Reprint requests may be directed to the author at Cognitive Rehabilitation Services, 38-25 52nd St., Sunnyside, NY 11104-1027.
20 min of testing time and runs on an ordinary IBMcompatible personal computer. Accordingly, the EDS is characterized as elemental in two ways: (1) its technical simplicity, and (2) its simulation of the elements of driving-related cognitive abilities.

\section{METHOD}

\section{Hardware}

The EDS, shown in Figure 1, consists of an IBM-compatible personal computer, software, a switch interface that accesses the computer through the game port, a 10-in.-diam steering wheel controlling a $150-\mathrm{k} \Omega$ linear potentiometer with turn signal (momentaryoff-momentary toggle switch with custom baton extension) and a heavy-duty momentary contact foot pedal. The software is written in compiled QuickBASIC and is fully menu driven. Timing is independent of the speed of the computer and is accurate to hundredths of a second. Apart from the computer, the EDS costs about $\$ 2,000$, and it includes a full-day training seminar.

\section{Software}

The assessment protocol begins with a self-appraisal of cognitive abilities related to driving: steering control, speed of reaction, self-control (impulsivity), field of view, consistency, and adjustment to changes and complexity. Each of these areas is then assessed in three increasingly complex simulated driving (steering) tasks (Phases 1-3). In each phase, the individual is allowed unlimited practice and decides when to proceed to the "test run." The rationale for this is that, since driving is an overlearned skill, assessment should minimize learning and emphasize practiced performance. Phase 1 is a preview tracking task that involves "steering" one's simulated "vehicle," which moves at a fixed pace in the center position of the driving lane. The road and vehicle are represented by symbols in 40 -column text mode-that is, the display is graphically simple, or elemental. Measures of lateral position are taken eight times per second and are stored for later analysis and reporting. In Phase 2, a two-choice reaction time task is added to the steering task, so that while the road advances, a small (one character subtending a visual angle of approximately $4^{\prime}$ ) stimulus face appears unpredictably on either side of the road. The individual must turn the signal lever on the steering column toward the face as soon 


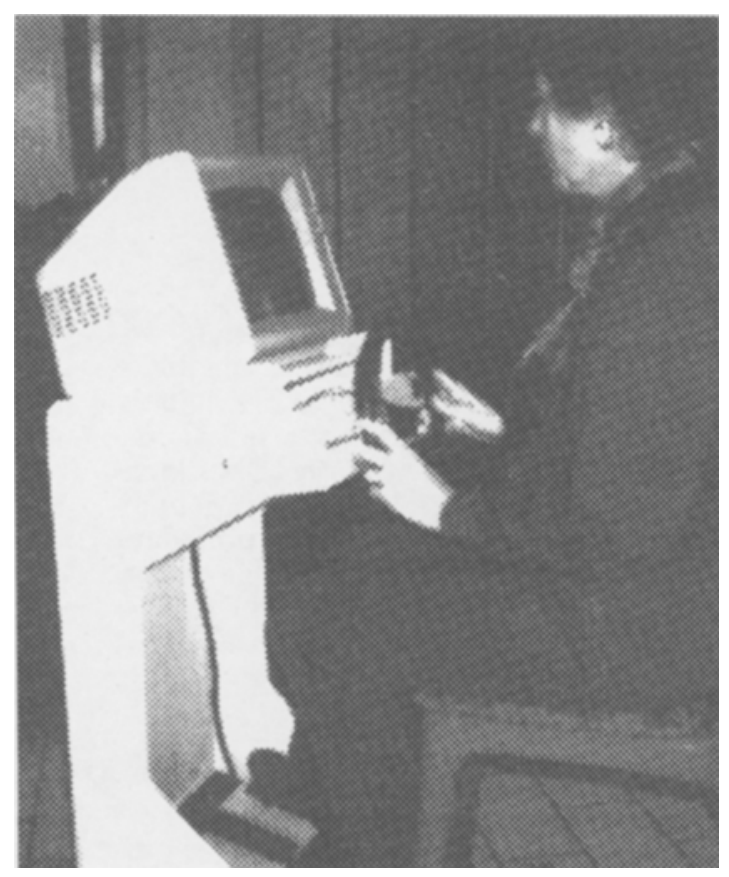

Figure 1. The free-standing model of the Elemental Driving Simulator (EDS). Not shown is the PC and switch interface box.

as possible, while maintaining a steady position in the center of the road. Reaction times are obtained and stored along with the steering measures.

In Phase 3, a contingency is introduced into the reaction time task: when the face is flashing (a "hazard"), the individual is to signal away from the face; otherwise, the signal is to be toward the face.

Results are saved to disk for research purposes and are presented in two printed reports: a technical report and a personal report in simple language for the examinee. Both reports include norm-based score summaries ( $M=100, S D=15)$, paired with self-appraisal ratings converted to the same scale.

\section{Case Example}

The case example that follows is intended to elaborate the clinical application of the EDS. This 55-year-old man sustained a severe traumatic brain injury in a motor vehicle accident 3 years ago and has been receiving cognitive rehabilitation therapy from the author for more than 2 years. He has been both eager and confident about driving throughout his recovery, but because of gross deficits in his attentional, visual perceptual, social, behavioral, and selfregulatory functions (not to mention his metacognition), formal assessment was deferred for 18 months. Interim mobility therapies focused on independence by foot and rail, and these were emented by an ongoing program of cognitive exercises and counseling.

He accepted the requirement that progress to an on-road evaluation was contingent upon performance within acceptable limits (cutoff of 2 standard deviations below the norm, or approximately the third percentile) on the EDS. He failed to complete the EDS on his first attempt. Retesting opportunities were scheduled at least a month apart, and he was offered opportunities to practice the steering component of the task. On his fourth attempt he succeeded, as illustrated in his Personal Report, which is shown in Figure 2.

My clinical summary of concerns cited the following: (1) simultaneous information processing; (2) ability to sustain attentional focus secondary to fatigue and distraction; (3) psychomotor co- ordination; (4) judgment-improved, but still overestimates; and (5) complex visual perception-sometimes exhibits a look-no-see reaction.

The next step was a rigorous road test given by two experienced driver-rehabilitation specialists in a range of driving environments. Their observations were recently confirmed by the present author and a driving instructor, on a 1.5-h drive. All the observers noted lane drifting, poor steering control, the participant's failure to turn his head to check the extreme left and right lanes, impulsivity, and difficulty in making adjustments. These observations were remarkably consistent with conclusions based on the EDS alone. Selfappraisal, though improved, remains inflated. Clinically, this case is ongoing; however, the EDS has served its most important purpose in guiding the individual's transition to behind-the-wheel assessment and training.

\section{Research Participants}

Research to date includes data from 50 normative drivers ( $M=$ 41 years), 1,145 older drivers from the community ( $M=69$ years), and 82 persons ( $M=37$ years) seeking driver rehabilitation because of a central nervous system disorder-traumatic brain injury $(n=28)$, cerebrovascular accident $(n=14)$, or cerebral palsy and other developmental neurological conditions $(n=25)$. The older drivers were volunteers in a large research project sponsored by the American Association of Retired Persons (AARP) and the ITT Hartford Insurance Company. Some had at-fault accidents on record, although specific information is not available. Most of the individuals in the rehabilitation sample were experienced drivers, except for those with developmental disabilities, who were learners.

The rehabilitation sample received a comprehensive driving evaluation at Gaylord Hospital in Wallingford, CT, where the therapists conduct hundreds of such evaluations each year. The evaluation included a medical and driving history, vision screening, the EDS, and, when deemed safe, a road test. Several other procedures, including the Doron Simulator, were used as needed. A pass or fail decision was made about each individual.

\section{RESULTS}

All the members in the normal group completed the three phases of the EDS; however, not all of the members in the other groups were able to do so. Representative box and whisker plots are shown in Figure 3. Each whisker shows a 1.96 standard error confidence interval; consequently, the groups whose means fall outside the others' whiskers are statistically distinct. Steering unsteadiness was indexed by the average change in relative road position in time samples that occurred approximately eight times per second. When an error occurred, the trial was presented again later. Reaction times (for correct trials) are reported for Phases 2 (simple) and 3 (complex, based on a binary contingency). The percent errors occurring in Phases 2 and 3 is taken as an index of impulsivity, although other cognitive factors (e.g., confusion) could be contributory.

The normal group's performance was more consistent and substantially better than that of the other groups. The true differences are probably even greater than they appear in Figure 3, because these data exclude the members of the rehabilitation and AARP samples who did not complete the EDS and who probably would have scored very poorly. As a group, those who failed were reliably worse than those who passed, especially on steering unsteadiness and complex reaction time. As a group, the 


\section{Elemental Driving Simulator: Personal Report}

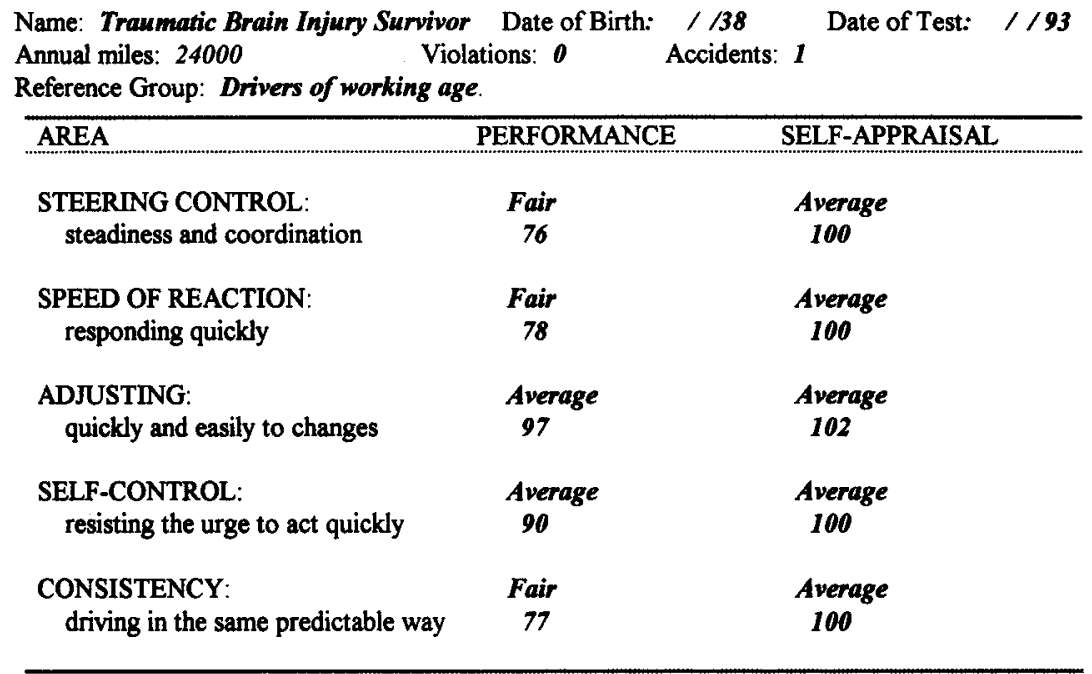

The 'SELF-APPRAISAL' column reflects how you predicted you would do. Your 'PERFORMANCE' rating is based on how you did compared to the scores obtained from a group of drivers of all ages.

An 'AVERAGE' rating ranges from 85 to 115 .

** Below 70 is UNACCEPTABLE: very few safe drivers score that low.

PERFORMANCE VS SELF-APPRAISAL (PSA) INDEX:-16.80 (BELOW self-estimates)

Your 'PERFORMANCE VS SELF-APPRAISAL INDEX' tells whether you did as well as you thought you would. A negative PSA index means you were over-confident.

Figure 2. EDS Personal Report for a survivor of traumatic brain injury. Self-appraisals and performance measures have been converted to a scale with an average of 100 and a standard deviation of 15. This report represents his fourth attempt. On the basis of the fact that all the measures were above the cutoff of 2 standard deviations for acceptability, he was recommended for behind-the-wheel testing.

older drivers (AARP volunteers) performed almost as poorly as the drivers who failed on all measures but impulsivity (i.e., errors in Phases 2 and 3), where they made fewer errors than both of the rehabilitation subgroups.

\section{DISCUSSION}

Overall, these findings substantiate the feasibility, reliability, and discriminative validity of the EDS procedure. Efficacy, however, must go beyond the statistical psychometric domain, and it is here that the simulation properties of the EDS are most helpful. It is reasonable to assume that the more realistic the simulation, the more convincing the results and the more effective the drivingadvisement process. The ultimate in realism is actual invehicle, behind-the-wheel, on-road testing (road tests), which probably accounts for their popularity. Even if one could contain the expense and, sometimes, the risk of road tests, one would have to address the serious criticism that road tests lack the basic psychometric requisites of testsstandardization, reliability, and empirical validity. Hence, realism, for all its clinical value, is not sufficient. Simulation allows us to have our cake (realism) and eat it too (empirical reliability and validity).

The issue then becomes: is the elemental realism of the EDS sufficient? There is no simple answer to this question. It is possible that the EDS, which people often find difficult, is sufficient to identify individuals who are at acceptable levels of risk, whereas those who do poorly on the EDS may require more elaborate simulation technologies and, possibly, on-road testing. In other words, the EDS may serve to screen for those who retain good skills, while indicating a need for additional diagnostic procedures in other cases.

In the longer run, components of the EDS will be enhanced by technologies now on the horizon, such as interactive video (see, e.g., Schiff \& Oldak, 1993), which will permit cognitively at-risk drivers to experience realistic driving scenarios. Like the EDS, the selection and design of these scenarios should systematically address the cognitive and metacognitive abilities that are needed for safe driving. 

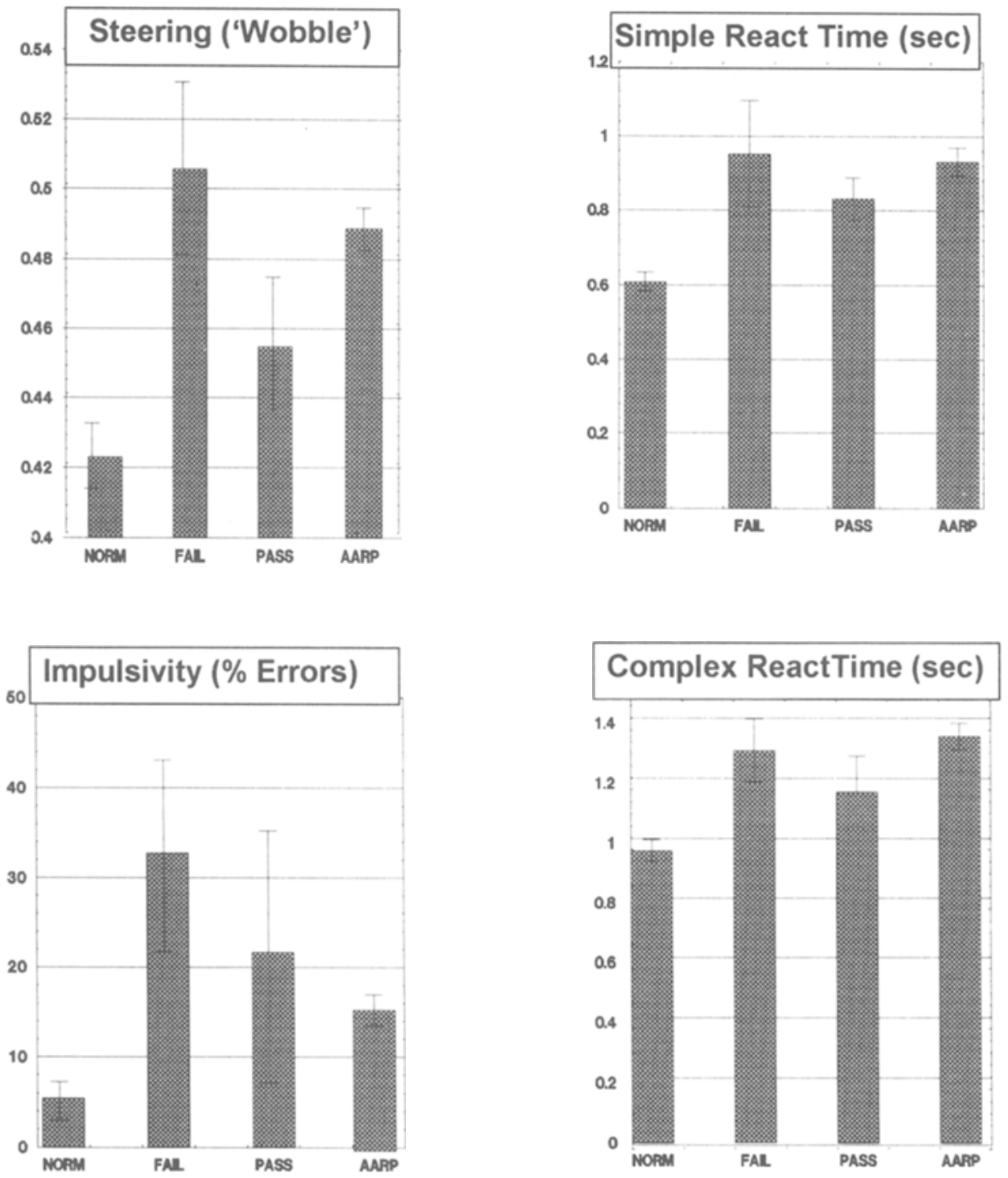

Figure 3. Performance on EDS measures by 1,276 persons from four subgroupings. NORM = drivers without clinical diagnosis of working age; FAIL = failed comprehensive rehabilitative driver evaluation, including on-road assessment when judged safe; PASS = passed comprehensive rehabilitative driver evaluation; and AARP = older American Association of Retired Persons members currently driving and participating as research volunteers. Each whisker represents a 1.96 standard error confidence interval, and group mean differences are likely to be statistically reliable if one mean falls outside the spread of the other. Steering "wobble" (upper left) is measured by the average absolute change in lateral vehicle position on the road from one time sample to the next. The units are essentially arbitrary and must be interpreted by reference to the normals' performance. Impulsivity (lower left) is indexed by the average percent errors on Phases 2 and 3. Simple reaction time (upper right) is the average response time in seconds for correct trials in Phase 2; complex reaction time (lower right) is for Phase 3.

\section{REFERENCES}

Gianutsos, R. G., Campbell, A., Beattie, A., \& Mandriota, F. (1992). The Driving Advisement System: A computer-augmented quasi-simulation of the cognitive prerequisites for resumption of driving after brain injury. Assistive Technology, 4, 70-86.

SCHIFF, W., \& OLDAK, R. (1993). Functional screening of older drivers using interactive computer-video scenarios. Washington, DC: AAA Foundation for Traffic Safety. 